\title{
INVERSE SCATTERING FOR AN EXTERIOR DIRICHLET PROBLEM*
}

\author{
BY
}

\section{S. I. HARIHARAN}

ICASE, NASA Langley Research Center, Hampton, Virginia 23665

\begin{abstract}
In this paper we consider scattering due to a metallic cylinder which is in the field of a wire carrying a periodic current. The aim of the paper is to obtain information such as the location and shape of the cylinder with a knowledge of a far-field measurement in between the wire and the cylinder. The same analysis is applicable in acoustics in the situation that the cylinder is a soft-wall body and the wire is a line source. The associated direct problem in this situation is an exterior Dirichlet problem for the Helmholtz equation in two dimensions. We present an improved low-frequency estimate for the solution of this problem using integral equation methods, and our calculations on inverse scattering are accurate to this estimate. The far-field measurements are related to the solutions of boundary integral equations in the low-frequency situation. These solutions can be expressed in terms of mapping function which maps the exterior of the unknown curve onto the exterior of a unit disk. The coefficients of the Laurent expansion of the conformal transformations can be related to the far-field coefficients. The first far-field coefficient leads to the calculation of the distance between the source and the cylinder. The other coefficients are determined by placing the source in a different location and using the corresponding new far-field measurements.
\end{abstract}

Introduction. In this note we are interested in the scattering due to a metallic cylinder which is in a field caused by a wire carrying a periodic current. The goal is to obtain information on the location and shape of the scatterer from a knowledge of the far field. A similar problem but with plane wave incidence has been recently studied by Colton [1] and Colton and Kleinmann [2]. A rigorous analysis on forward scattering in this situation has been recently given by Hariharan and MacCamy [3]. If the metal is not made of ferromagnetic material then one will obtain an interior-exterior problem with the boundary of the conductor as an interface. However, for a ferromagnetic conductor one will obtain an exterior Dirichlet problem. The details of this discussion are available in [3]. The analysis in what follows is also applicable to acoustic wave propagation in the situation where the scatterer is a soft material. The precise model of interest is illustrated in Fig. 1. and 2.

Let $\Omega$ be the cross-section of the cylinder in the $x-y$ plane, $\Omega^{+}$its exterior and $\Gamma$ its boundary. The wire which is located at $\mathbf{x}_{0} \in \Omega^{+}$carries a periodic current of the form

\footnotetext{
* Received September 23, 1981. This research was supported by NASA Contracts No. NAS1-16394 and NAS1-15810 while the author was in residence at ICASE, NASA Langley Research Center, Hampton, VA 23665. The author is greatly indebeted to Professor D. L. Colton for his interest in this work and his suggestions and encouragement. Discussions with Professor W. D. Lakin are also greatly appreciated.
} 

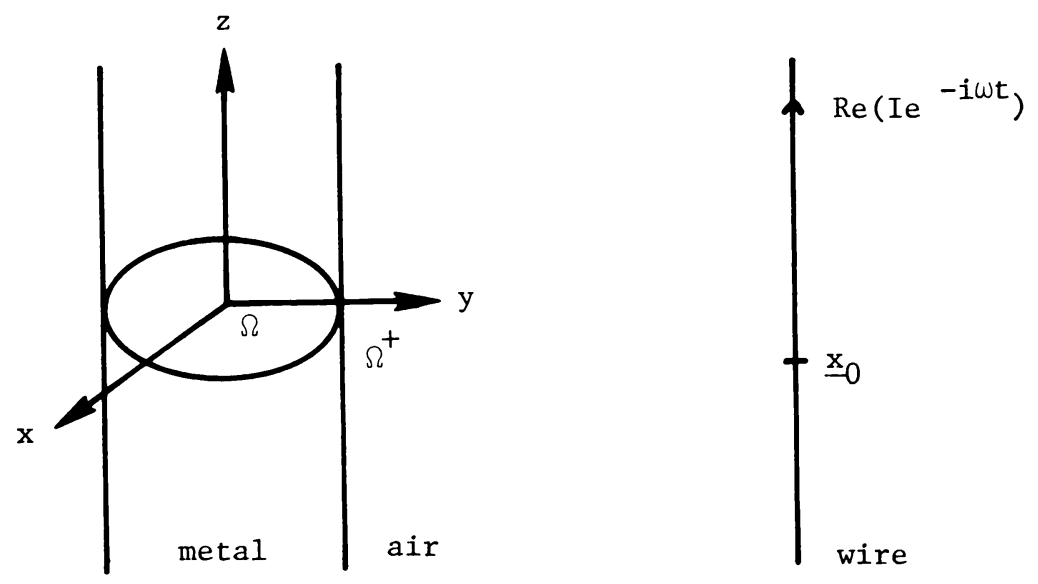

FIG. 1.

$\operatorname{Re}\left(I e^{-i \omega t}\right)$. The derivation of the associated general problem is discussed in [3]. For details we refer the reader to this paper and present here the particular problem. Find $u \in$ $C^{2}\left(\Omega^{+} \backslash\left\{\mathbf{x}_{0}\right\}\right) \cup C^{1}(\Gamma)$ such that

$$
\begin{aligned}
& u-u_{\beta} \in C^{2}\left(\Omega^{+}\right) \bigcup C^{1}(\Gamma), \\
& \Delta u+\beta^{2} u=0 \text { in } \Omega^{+}, \\
& u=0 \text { on } \Gamma,
\end{aligned}
$$

$$
u-u_{\beta} \text { satisfies the Sommerfeld radiation condition. }
$$

Here $u_{\beta}$ is the potential due to the wire and is prescribed by

$$
u_{\beta}=-\frac{i}{4} H_{0}^{(1)}\left(\beta\left|\mathbf{x}-\mathbf{x}_{0}\right|\right) \quad\left(\mathbf{x} \in \Omega^{+}\right),
$$

and $\beta$ is a non-dimensional parameter which depends on the frequency $\omega$. For moderate frequencies such as household currents or AM transmissions the value of $\beta$ ranges from $10^{-9}<\beta<10^{-2}$. This is the size of $\beta$ which is of interest in this note. Since $\beta^{2}$ is extremely small one might take $\beta=0$ in problem I, but there are two difficulties: first, the radiation condition will no longer be valid and second, the incident field potential (1.1) will become logarithmically infinite. However, it turns out that the following theorem is true.

THEOREM 1.1. $u=v+O(1 / \log \beta)$ as $\beta \rightarrow 0$, where $v$ satisfies the following problem:

$$
\begin{aligned}
v & \in C^{2}\left(\Omega^{+} \backslash\left\{\mathbf{x}_{0}\right\}\right) \bigcup C^{1}(\Gamma), \\
v-v_{0} & \in C^{2}\left(\Omega^{+}\right) \bigcup C^{1}(\Gamma), \\
\Delta v & =0 \quad \text { in } \Omega^{+}, \\
v & =0 \quad \text { on } \Gamma,
\end{aligned}
$$

$v$ bounded as $|\mathbf{x}| \rightarrow \infty \quad\left(\mathbf{x} \in \mathbb{R}^{2}\right)$, 


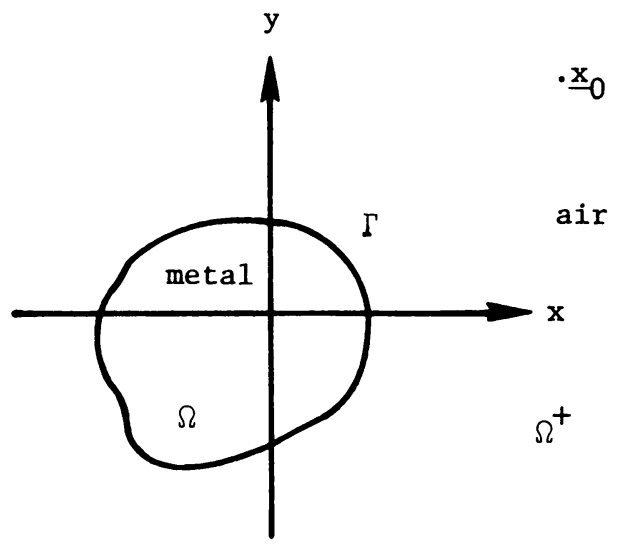

FIG. 2.

where

$$
v_{0}(\mathbf{x})=\frac{1}{2 \pi} \log \left|\mathbf{x}-\mathbf{x}_{0}\right| \quad\left(\mathbf{x} \in \Omega^{+}\right) .
$$

Thus the solution of problem II will be the limiting value of the solution of problem I as $\beta \rightarrow 0$.

In order to solve the inverse scattering problem several details of forward scattering are required. In this process we shall prove Theorem 1.1 and also provide an improved estimate.

THEOREM 1.2.

$$
u=v+\frac{C F_{0}}{\log \beta+\delta}+O\left(\beta^{2} \log \beta\right)
$$

where $C \in \mathbb{R}, \delta \in \mathbb{C}$ and $F_{0} \subset C^{2}(\Omega) \cup C^{1}(\Gamma)$ is a uniquely determined function.

Hence our results on inverse scattering would allow one to obtain the shape of the boundary for low frequencies accurate to $O\left(\beta^{2} \log \beta\right)$.

2. Forward scattering. Here we shall indicate the solution procedure for problems I and II if the boundary, $\Gamma$ (sufficiently smooth), is given. We shall use simple layer potentials for the construction of our solutions.

Let

where

$$
\mathscr{U}_{\delta}[\sigma](\mathbf{x})=\int_{\Gamma} \sigma(\mathbf{y}) G_{\delta}(\mathbf{x}, \mathbf{y}) d s_{\mathbf{y}} \quad(\delta \in \mathbb{R})
$$

$$
G_{\delta}(\mathbf{x}, \mathbf{y})= \begin{cases}\frac{1}{2 \pi} \log |x-y| & \text { if } \delta=0 \\ -\frac{i}{4} H_{0}^{(1)}(\delta|\mathbf{x}-\mathbf{y}|) & \text { if } \delta \neq 0\end{cases}
$$


Then the following properties hold:

(i) $\Delta \mathscr{U}_{\delta}+\delta^{2} \mathscr{U}_{\delta}=0$ in $\mathbb{R}^{2}$,

(ii) $\mathscr{U}_{\delta} \in C\left(\mathbb{R}^{2}\right)$,

(iii) $\mathscr{U}_{\delta}$ satisfies Sommerfeld's radiation condition if $\delta \neq 0$,

(iv) $\mathscr{U}_{\delta} \sim\left(\int_{\Gamma} \sigma(\mathbf{y}) d s_{\mathbf{y}}\right) \log |\mathbf{x}|$ as $|\mathbf{x}| \rightarrow \infty$ if $\delta=0$.

Notation:

$$
\mathscr{U}_{\delta}[\sigma](\mathbf{x})=\mathscr{V}_{\delta}[\sigma](\mathbf{x}) \quad \text { for } \mathbf{x} \in \Gamma .
$$

We shall use this notation throughout this section.

Thus one can seek a solution of problem I in the form

$$
u(\mathbf{x})=\mathscr{U}_{\beta}[\phi](\mathbf{x})+u_{\beta}(\mathbf{x}) \quad\left(\mathbf{x} \in \Omega^{+}\right) .
$$

Then (2.1) will be a solution of (I) provided

$$
\mathscr{V}_{\beta}[\phi]+u_{\beta}=0 \quad \text { on } \Gamma \text {. }
$$

Similarly, we seek the solution of problem (II) in the form of a simple layer except incorporating a constant $C \in \mathbb{R}$ which is:

$$
v(\mathbf{x})=\mathscr{U}_{0}[\psi](\mathbf{x})+v_{0}(\mathbf{x})+C \quad\left(\mathbf{x} \in \Omega^{+}\right) .
$$

Demanding that $v$ satisfies the boundary condition and the condition at infinity, we have

$$
\mathscr{V}_{0}[\psi]+v_{0}+C=0 \quad \text { on } \Gamma, \quad \int_{\Gamma} \psi(s) d s=-1 .
$$

Remark 2.1. (a) The constant $C$ is incorporated in (2.2) in order to make the system of integral equations (IV) consistent. This is a standard argument. (b) The uniqueness of problems I and II are also standard, as is the existence of $\phi$ and $(\psi, C)$ (see for example [3, 4]). Our low-frequency theory is based on the existence proofs presented in [3].

To present our main result we need several lemmas. We shall begin by stating them. (The proofs may be found in [3].)

Lemma 2.1. There exists a unique solution $\left(f_{0}, \Gamma_{0}\right) \subset C(\Gamma) \times \mathbb{R}$ for the system of integral equations

$$
\mathscr{V}_{0}\left[f_{0}\right]=\Gamma_{0}, \quad \int_{\Gamma} f_{0}(s) d s=1 .
$$

Moreover, the solution is given by

$$
f_{0}=-\frac{1}{2 \pi} \frac{\partial}{\partial n} \log |f(z)|, \quad \Gamma_{0}=\frac{1}{2 \pi} \log |f(z)| \quad(z \in \Gamma)
$$

where $\omega=f(z)$ is the unique mapping function which maps $\Gamma$ on to the exterior of a unit disk $|\omega|=R$ such that $f(z) \sim z$ as $z \rightarrow \infty$.

Remark 2.2. If we map $\Gamma$ onto the exterior of a unit disk with $f(z) \sim a z$ as $z \rightarrow \infty(a>0$, $a^{-1}$ is the mapping radius) then $|\omega|=|f(z)|=1$. Thus $\Gamma_{0}=0$.

As an aside, we would like to point out that the result of this lemma enabled us in [3] to prove the existence of the system without the condition that the mapping radius should not equal one. This is different than the proof available in [4]. The following two lemmas may also be found in [3]. A version of these proofs with fewer regularity assumptions are available in [5]. 
LEMMA 2.2. There exists a kernel $P(s, \sigma)$ of the form

$$
P(s, \sigma)=-\frac{1}{\pi} \cot \frac{s-\sigma}{2}+R(s, \sigma),
$$

with $R(\cdot, \cdot)$ analytic such that

$$
f(s)=f_{P}(s):=\int_{0}^{2 \pi} P(s, \sigma) h^{\prime}(\sigma) d \sigma,
$$

satisfies

$$
\mathscr{V}_{0}[f](s)=h(s)+\Gamma(h)
$$

where $\Gamma(h)$ (a constant) is a functional of $h$ given by

$$
\Gamma(h)=\frac{1}{2 \pi}\left\{\int_{0}^{2 \pi} \mathscr{V}_{0}\left[f_{p}\right](\sigma) d \sigma-\int_{0}^{2 \pi} h(\sigma) d \sigma\right\} .
$$

Remark 2.3. Let us observe for later purposes that when $h \equiv 1$

$$
\Gamma(1)=-1, \quad f_{p}=0 .
$$

It is shown in [3] that the combination of Lemma 2.1 and 2.2 yields the existence of (III). In particular, when we set $h(s)=-v_{0}(s)$ we have the following lemma.

LEMma 2.3. The unique solution of the system IV is given by

$$
\begin{gathered}
\psi=f_{p}+\alpha f_{0}, \\
C=-\Gamma(h)-\alpha \Gamma_{0},
\end{gathered}
$$

where $\alpha \in \mathbb{R}$ is a functional of $h$ given by

$$
\alpha(h)=-1-\int_{\Gamma} f_{P}(s) d s .
$$

Remark 2.4. In Lemma 2.2 the normalization of $\Gamma$ over $[0,2 \pi]$ is used for convenience.

Now we return to integral equation III. First of all, by the expansion of the Hankel function $H_{0}^{(1)}(z)$ for small $z$ we have

$$
-\frac{i}{4} H_{0}^{(1)}(z)=\frac{1}{2 \pi}\{\log (z / 2)+(\gamma-(\pi / 2) i)\}+O\left(z^{2} \log z\right) .
$$

Using this equation (III) for small $\beta$ leads to the following equation:

$$
\mathscr{V}_{0}[\phi]+m \int_{\Gamma} \phi(s) d s+v_{0}+m+O\left(\beta^{2} \log \beta\right)=0,
$$

where

$$
m=\frac{1}{2 \pi}\left\{\log \left(\frac{\beta}{2}\right)+\gamma-\frac{\pi}{2} i\right\}
$$


In (2.13) the term $O\left(\beta^{2} \log \beta\right)$ contains integral operators involving $\phi$. Motivated by (2.13), we consider an associated equation

$$
\mathscr{V}_{0}[\tilde{\phi}]+m \int_{\Gamma} \tilde{\phi}(s) d s+m=h,
$$

where $h=-v_{0}$. It will be argued later that

$$
\phi=\tilde{\phi}+O\left(\beta^{2} \log \beta\right) .
$$

LeMma 2.4. The solution of (III*) is given by

$$
\tilde{\phi}=\psi+C f_{0} /\left(\Gamma_{0}+m\right) .
$$

Proof. This is done in two steps. First we seek a solution of III* in the form $\tilde{\phi}=-m g_{1}$ $+g_{2}$, where $g_{1}$ and $g_{2}$ satisfy

$$
\begin{aligned}
& \mathscr{V}_{0}\left[g_{1}\right]+m \int_{\Gamma} g_{1}(s) d s=1, \\
& \mathscr{V}_{0}\left[g_{2}\right]+m \int_{\Gamma} g_{2}(s) d s=h .
\end{aligned}
$$

We look for the solution of $(2.15)$ in the form

$$
g_{2}=f_{P}+\lambda f_{0} \quad(\lambda \in \mathbb{C}) .
$$

Demanding that $g_{2}$ satisfy (2.15), we have

$$
\mathscr{V}_{0}\left[f_{P}\right]+\lambda \mathscr{V}_{0}\left[f_{0}\right]+m \int_{\Gamma} f_{P}(s) d s+\lambda m \int_{\Gamma} f_{0}(s) d s=h,
$$

and using Lemmas 2.1 and 2.2 we have

$$
\lambda=\lambda(h)=\frac{-\Gamma(h)-m \int_{\Gamma} f_{P}(s) d s}{m+\Gamma_{0}} .
$$

Thus $g_{2}$ is known. The solution of (2.14) is obtained in the same way setting $h=1$ :

$$
g_{1}=f_{P}+\lambda(1) f_{0} \text {. }
$$

We observe by Remark (2.3) that

$$
\lambda(1)=\frac{1}{m+\Gamma_{0}}, \quad g_{1}=\lambda(1) f_{0},
$$

and hence

$$
\tilde{\phi}=f_{P}+(\lambda(h)-m \lambda(1)) f_{0}=f_{P}+\frac{-\Gamma(h)-\left(1+\int_{\Gamma} f_{P}(s) d s\right) m}{m+\Gamma_{0}} f_{0} .
$$

Using the definition of $\alpha(h)$ in (2.12), we have

or

$$
\begin{gathered}
\tilde{\phi}=f_{P}+\frac{-\Gamma(h)-\Gamma_{0} \alpha+\left(m+\Gamma_{0}\right) \alpha}{m+\Gamma_{0}} f_{0}, \\
\tilde{\phi}=f_{P}+\alpha f_{0}-\frac{\Gamma(h)+\Gamma_{0} \alpha}{m+\Gamma_{0}} f_{0} .
\end{gathered}
$$


Now using (2.10) and (2.11) we have

$$
\tilde{\phi}=\psi+\frac{C f_{0}}{m+\Gamma_{0}},
$$

and the lemma.

Remark 2.5. Uniqueness of the solution III* can be argued by considering the homogeneous equation of III*.

Now we shall return to our main theorems, 1.1 and 1.2. We shall describe the proof of Theorem 1.2 which also proves Theorem 1.1. First of all we indicate the argument that

$$
\phi=\tilde{\phi}+O\left(\beta^{2} \log \beta\right) \text {. }
$$

To do this we simply consider the difference of Eqs. (III) and (III*), which is

$$
\mathscr{V}_{0}[\phi-\tilde{\phi}]+m \int_{\Gamma}(\phi-\tilde{\phi})(s) d s=O\left(\beta^{2} \log \beta\right) .
$$

The operator on the left-hand side is invertible. This can be shown in the same way we did for Eq. (2.15) with $h=O\left(\beta^{2} \log \beta\right)$, and the inverse operations are performed on terms of order $O\left(\beta^{2} \log \beta\right)$. Thus we have

$$
\phi=\tilde{\phi}+O\left(\beta^{2} \log \beta\right) .
$$

Now we return to the simple layer representation (2.1)

$$
u(\mathbf{x})=\mathscr{U}_{\beta}[\phi](\mathbf{x})+u_{\beta}(\mathbf{x}) .
$$

From the expansion of Hankel function for small $\beta$ we have

$$
u(\mathbf{x})=\mathscr{U}_{0}[\phi](\mathbf{x})+m \int_{\Gamma} \phi(s) d s+m+v_{0}+O\left(\beta^{2} \log \beta\right) .
$$

Substituting the relation $(2.20)$ we have

$$
u(\mathbf{x})=\mathscr{U}_{0}[\tilde{\phi}](\mathbf{x})+m \int_{\Gamma} \tilde{\phi}(s) d s+m+v_{0}+O\left(\beta^{2} \log \beta\right),
$$

and hence from Lemma 2.4 we have

$$
\begin{aligned}
u(\mathbf{x})=\mathscr{U}_{0}[\psi](\mathbf{x})+\frac{C}{m+\Gamma_{0}} \mathscr{U}_{0}\left[f_{0}\right](\mathbf{x})+m & \int_{\Gamma} \psi(s) d s \\
& +\frac{C m}{m+\Gamma_{0}} \int_{\Gamma} f_{0}(s) d s+m+v_{0}(\mathbf{x})+O\left(\beta^{2} \log \beta\right) .
\end{aligned}
$$

But

$$
\int_{\Gamma} \psi(s) d s=-1, \quad \int_{\Gamma} f_{0}(s) d s=1 .
$$

Thus,

$$
u(\mathbf{x})=\mathscr{U}_{0}[\psi](\mathbf{x})+\frac{C}{m+\Gamma_{0}} \mathscr{U}_{0}\left[f_{0}\right](\mathbf{x})+\frac{C m}{m+\Gamma_{0}}+v_{0}(\mathbf{x})+O\left(\beta^{2} \log \beta\right) .
$$

Observing that

$$
\frac{C m}{m+\Gamma_{0}}=C-\frac{\Gamma_{0} C}{m+\Gamma_{0}},
$$


we have

$$
u(\mathbf{x})=\mathscr{U}_{0}[\psi](\mathbf{x})+v_{0}(x)+C+\frac{C}{m+\Gamma_{0}}\left\{\mathscr{U}_{0}\left[f_{0}\right](\mathbf{x})-\Gamma_{0}\right\}+O\left(\beta^{2} \log \beta\right) .
$$

From the simple layer potential for problem (II) we have

$$
u(\mathbf{x})=v(\mathbf{x})+\frac{C}{m+\Gamma_{0}}\left\{\mathscr{U}_{0}\left[f_{0}\right](\mathbf{x})-\Gamma_{0}\right\}+O\left(\beta^{2} \log \beta\right) .
$$

This proves Theorem 1.2 and Theorem 1.1 follows immediately.

3. Far-field measurements and inverse scattering. In this section we follow the work appearing in [1] and [2]. The main idea is to obtain a sequence of moment problems relating the Fourier coefficients of the far field to the coefficients of the Laurent expansions of the conformal transformation which maps the exterior of a circle onto the exterior of the unknown curve. As in [1] and [2], it turns out that these moment problems are solvable in terms of the mapping radius $a$, which in turn is determined by placing the source in a different position. The main difference here is that the location of the source can be calculated; thus one indeed finds the location of the scatterer. Such a result is useful in radar detection, where one is interested more in the location of the radar than the shape.

The physical situation is portrayed in Fig. 3. To avoid repetition we shall cite [1] or [2] wherever possible. We assume that the far-field measurements are available on a circle of radius $r$ and let the source be at a distance $\left|\mathbf{x}_{0}\right|$ such that $\left|\mathbf{x}_{0}\right|>r$. From problem I, the scattered part of $u$ is given by

$$
u^{s}(\mathbf{x})=-\frac{i}{4} \int_{\Gamma} \phi(\mathbf{y}) H_{0}^{(1)}(\beta|\mathbf{x}-\mathbf{y}|) d s_{\mathbf{y}} .
$$

From the asymptotic behavior of the Hankel function one has

$$
u^{s}(r, \theta)=\frac{1}{4} e^{i(\beta r+\pi / 4)} \sqrt{\frac{2}{\pi \beta r}} F(\theta ; \beta)+O\left(\frac{1}{r^{3 / 2}}\right),
$$

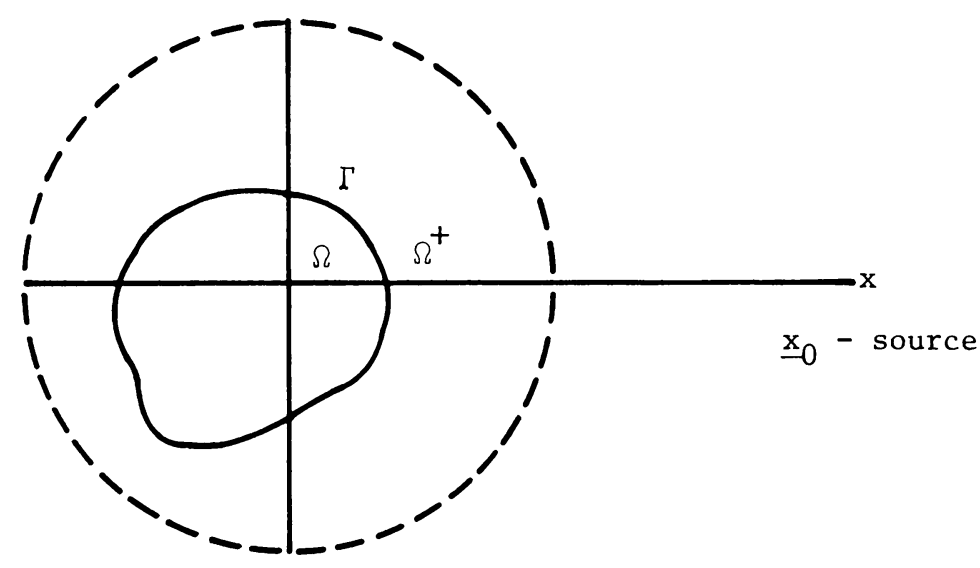

FIG. 3. 
where $\mathbf{x}=(r, \theta)$ and $\mathbf{y}=(\rho, \phi)$ in polar coordinates and

$$
F(\theta ; \beta)=-\int_{\Gamma} \phi(\mathbf{y}) e^{-i \beta \rho \cos \overline{\theta-\phi}} d s_{\mathbf{y}} .
$$

$F$ is known as the far field. Expanding $F(\theta ; \beta)$ in a Fourier series, we have

$$
F(\theta ; \beta)=\sum_{n=-\infty}^{\infty} a_{n}(\beta) e^{i n \theta}
$$

where

$$
\begin{aligned}
a_{n}(\beta) & =-\frac{1}{2 \pi} \int_{-\pi}^{\pi} \int_{\Gamma} \phi(\mathbf{y}) e^{-i n \theta-i \beta \rho \cos \overline{\theta-\phi}} d s_{\mathbf{y}} d \theta \\
& =-i^{-n} \int_{\Gamma} \phi(\mathbf{y}) J_{n}(\beta \rho) e^{-i n \phi} d s_{\mathbf{y}} .
\end{aligned}
$$

Remark 3.1. Usually one measures a finite number of $a_{n}$ 's. We shall be concerned only with $a_{n}$ for $n \geq 0$. Negatively indexed coefficients do not play a role in our calculation (see [2]).

The Taylor series expansion of Bessel's function in (3.3) yields

$$
a_{n}(\beta)=\frac{-i^{-n} \beta^{n}}{2^{n} n !} \int_{\Gamma} \phi(\mathbf{y}) \rho^{n} e^{-i n \phi} d s_{\mathbf{y}} .
$$

Define

$$
\mu_{n}(\beta)=2^{n} n ! a_{n}(\beta) i^{n} / \beta^{n}
$$

Therefore

$$
\mu_{n}(\beta)=-\int_{\Gamma} \phi(\mathbf{y}) \rho^{n} e^{-i n \phi} d s_{\mathbf{y}}
$$

Now using (2.20) we have

$$
\mu_{n}(\beta)=-\int_{\Gamma} \tilde{\phi}(y) \rho^{n} e^{-i n \phi} d s_{\mathbf{y}} \quad\left(\bmod \left(O\left(\beta^{2} \log \beta\right)\right) .\right.
$$

We shall drop $\bmod \left(O\left(\beta^{2} \log \beta\right)\right)$ for convenience of writing. Observe that from Lemma 2.4 we have

$$
\tilde{\phi}(\mathbf{y})=\psi(\mathbf{y})+\frac{C f_{0}}{m+\Gamma_{0}}
$$

and from the simple layer potential $(2.2), \psi(\mathbf{y})$ is given by

$$
\psi(\mathbf{y})=-\frac{\partial}{\partial n}\left(v-v_{0}\right)(\mathbf{y}), \quad \mathbf{y} \in \Gamma
$$

where the normal derivative is in the outward direction. We want to map $\Gamma$ onto the exterior of a unit circle by

$$
\omega=f(z)=a z+O(1)
$$

where $a$ is the mapping radius. Thus $\Gamma_{0}=0$. We would also like to obtain $\psi(z)$ in terms of this mapping function $f(z)$. For this we have: 
LemMA 3.1. Let $\omega=f(z)=a z+O(1)$ be the unique mapping function which maps $\Gamma$ onto the exterior of the unit circle $|\omega|=1$. Then the solution of problem II is given by

$$
v(z)=\frac{1}{2 \pi} \log \left|f(z)-f\left(z_{0}\right)\right|-\frac{1}{2 \pi} \log \left|f(z) \bar{f}\left(z_{0}\right)-1\right| ;
$$

where $z_{0}$ is the location of the source and the bar denotes conjugation.

Proof. We make use of the uniqueness of the solution of problem II. First observe that the expression given by (3.7)

(i) satisfies Laplace's equation,

(ii) is bounded as $z \rightarrow \infty$ (since $f(z) \sim a z$ as $z \rightarrow \infty$ ), and

(iii) satisfies $v(z)=0$ for $z \in \Gamma$ (this follows from the fact that $1 / f(z)=\bar{f}(z)$ for $z \in \Gamma$ ),

(iv) furthermore, $v-v_{0}$ is regular (since $\log \left|f(z)-f\left(z_{0}\right)\right|$ has leading singularity log $\left.\left|z-z_{0}\right|\right)$.

We now consider the behavior of $v(z)-v_{0}(z)$ for $z_{0}$ large. First observe that from the Taylor series of $\log \left|z-z_{0}\right|$ for $\left|z_{0}\right|$ large

$$
v_{0}(z)=\frac{1}{2 \pi} \log \left|z-z_{0}\right| \simeq \frac{1}{2 \pi} \log \left|z_{0}\right| .
$$

Since $\left|z_{0}\right|$ is large, $f\left(z_{0}\right)=a z_{0}+b$ where $b$ has the form

$$
b=B_{0}+\frac{B_{1}}{z_{0}}+\frac{B_{2}}{z_{0}^{2}}+\ldots
$$

We want to use this in the expression (3.7) for $v$. First of all

$$
\begin{aligned}
\log \left|f(z)-f\left(z_{0}\right)\right| & =\log \left|f(z)-\left(a z_{0}+b\right)\right|=\log \left|a z_{0}\left(1-\frac{f(z)-b}{a z_{0}}\right)\right| \\
& =\log \left|a z_{0}\right|+\log \left|\left(1-\frac{f(z)-b}{a z_{0}}\right)\right| \sim \log \left|a z_{0}\right|-\text { R.P. }\left(\frac{f(z)-b}{a z_{0}}\right),
\end{aligned}
$$

where R.P. denotes the real part. Since $a>0, \bar{f}\left(z_{0}\right)=a \bar{z}_{0}+\bar{b}$. Therefore the second expression in (3.7) becomes

$$
\begin{aligned}
\log \left|f(z) \bar{f}\left(z_{0}\right)-1\right| & =\log \left|\left(a \bar{z}_{0}+\bar{b}\right) f(\bar{z})-1\right| \\
& =\log \left|a z_{0} f(z)\right|+\log \mid\left\{1-\frac{1}{a \bar{z}_{0}}\left(\frac{1}{f(z)}-\bar{b}\right)\right\} \\
& \sim\left\{\log \left|a z_{0}\right|+\log |f(z)|-\text { R.P. }\left(\frac{1}{a \bar{z}_{0}} \frac{1}{f(z)}-\bar{b}\right)\right\} .
\end{aligned}
$$

Thus, noting that R.P. $\left(\bar{b} / \bar{z}_{0}\right)=$ R.P. $(b / z)$, we have

$$
v(z) \sim-\frac{1}{2 \pi} \log |f(z)|-\frac{\text { R.P. }}{2 \pi a}\left(\frac{f(z)}{z_{0}}-\frac{1}{\bar{z}_{0}} f(z)\right) .
$$

We now consider two special cases.

Case $(i): z_{0} \equiv(\alpha, 0)=\alpha$ (source on $x$ axis). In this case

$$
v(z)=-\frac{1}{2 \pi} \log |f(z)|-\frac{1}{2 \pi a \alpha} \text { R.P. }\left(f(z)-\frac{1}{f(z)}\right) .
$$


Case (ii): $z_{0}=(0, \alpha)=i \alpha$ (source on $y$ axis). Then

$$
v(z)=-\frac{1}{2 \pi} \log |f(z)|-\frac{1}{2 \pi a \alpha} \text { I.P. }\left(f(z)+\frac{1}{f(z)}\right),
$$

where I.P. denotes the imaginary part. Therefore, (3.6) gives

$$
\psi(z)=-\frac{\partial}{\partial n}\left\{\begin{array}{l}
-\frac{1}{2 \pi} \log |f(z)|-\frac{1}{2 \pi a \alpha} \text { R.P. }\left(f(z)-\frac{1}{f(z)}\right) \\
-\frac{1}{2 \pi} \log |f(z)|-\frac{1}{2 \pi a \alpha} \text { I.P. }\left(f(z)+\frac{1}{f(z)}\right) \quad \text { for } \quad z_{0}=\alpha
\end{array}\right.
$$

First we consider the case that $z_{0}=\alpha$. In this case $\tilde{\phi}$ in (3.5) gives

$$
\tilde{\phi}(z)=\frac{1}{2 \pi a \alpha} \text { R.P. } \frac{\partial}{\partial n}\left(f(z)-\frac{1}{f(z)}\right)+\left(\frac{C}{m}+1\right) \frac{1}{2 \pi} \frac{\partial}{\partial n} \log |f(z)| .
$$

We use this in the expression for $\mu_{n}$ which is

$$
\mu_{n}=-\int_{\Gamma} \tilde{\phi}(z) \rho^{n} e^{-i n \phi} d s
$$

Taking complex conjugates

$$
\bar{\mu}_{n}=-\int_{\Gamma} \tilde{\phi}(z) \rho^{n} e^{\mathrm{in} \phi} d s,
$$

or using $z=\rho e^{i \phi}$ and (3.10), we have:

$$
\bar{\mu}_{n}=-\frac{1}{2 \pi a \alpha} \int_{\Gamma} \text { R.P. } \frac{\partial}{\partial n}\left(f(z)-\frac{1}{f(z)}\right) z^{n} d s-\frac{1}{2 \pi}\left(\frac{C}{\bar{m}}+1\right) \int_{\Gamma} \frac{\partial}{\partial n} \log |f(z)| z^{n} d s .^{\dagger}
$$

To deal with the integrals in (3.11) let us make the following observation:

$$
\frac{\partial}{\partial n} \text { R.P. }\left(f(z)-\frac{1}{f(z)}\right)=-\frac{\partial}{\partial s} \operatorname{Im}\left(f(z)-\frac{1}{f(z)}\right),
$$

by the Cauchy-Riemann equations.

But

$$
\text { R.P. }\left(f(z)-\frac{1}{f(z)}\right)=0 \quad \text { for } z \in \Gamma .
$$

Thus

$$
\frac{\partial}{\partial n} \text { R.P. }\left(f(z)-\frac{1}{f(z)}\right)=-\frac{\partial}{\partial s}\left(f(z)-\frac{1}{f(z)}\right) \text {. }
$$

Similarly,

$$
\frac{\partial}{\partial n} \log |f(z)|=-\frac{\partial}{\partial s} \arg f(z) .
$$

Since

$$
\omega=f(z), \quad z=f^{-1}(\omega),
$$


we can rewrite $(3.11)$ as

$$
\bar{\mu}_{n}=\frac{1}{2 \pi a \alpha} \int_{\Gamma} \frac{\partial}{\partial s}\left(\omega-\frac{1}{\omega}\right)\left[f^{-1}(\omega)\right]^{n} d s+\frac{1}{2 \pi}\left(\frac{C}{\bar{m}}+1\right) \int_{\Gamma} \frac{\partial}{\partial s} \arg \omega\left[f^{-1}(\omega)\right]^{n} d s
$$

Observing that

$$
\frac{\partial}{\partial s} \arg \omega d s=\frac{\partial}{\partial \omega} \arg \omega d \omega=-\frac{i}{\omega} d \omega \text { on }|\omega|=1
$$

(see [2] for these calculations) we have that (3.12) can be written in the form

$$
\bar{\mu}_{n}=\frac{1}{2 \pi a \alpha} \int_{|\omega|=1} \frac{\partial}{\partial \omega}\left(\omega-\frac{1}{\omega}\right)\left[f^{-1}(\omega)\right]^{n} d \omega-\frac{i}{2 \pi}\left(\frac{C}{\bar{m}}+1\right) \int_{|\omega|=1} \frac{1}{\omega}\left[f^{-1}(\omega)\right]^{n} d \omega .
$$

Since $f(z)$ has the Laurent expansion

$$
f(z)=a z+B_{0}+\frac{B_{1}}{z}+\frac{B_{2}}{z^{2}}+\ldots=\omega \quad\left(B_{i} \in \mathbb{C}, i=0,1,2, \ldots\right) .
$$

we have that $f^{-1}$ has a Laurent expansion of the form

$$
f^{-1}(\omega)=\frac{\omega}{a}+b_{0}+\frac{b_{1}}{\omega}+\frac{b_{2}}{\omega^{2}}+\ldots \quad\left(b_{i} \in \mathbb{C}, i=0,1,2, \ldots\right) .
$$

Since $\Gamma$ is smooth this series is uniformly convergent on $|\omega|=1$. We substitute (3.14) in (3.13) and evaluate the residues for each $n$. We shall indicate the first three and the rest can be done recursively. For simplicity we shall take $b_{0}=0$, since it represents just a translation (which translation will apply for both the origin of the scatterer and the location of the source).

$$
\begin{array}{lc}
\text { For } n=0: & \bar{\mu}_{0}=\frac{C}{\bar{m}}+1 . \\
\text { For } n=1: & \bar{\mu}_{1}=\frac{i}{a \alpha}\left(\frac{1}{a}+b_{1}\right) . \\
\text { For } n=2: & \bar{\mu}_{2}=\frac{2 i}{a \alpha} b_{2}+\left(\frac{C}{\bar{m}}+1\right) \frac{2 b_{1}}{a},
\end{array}
$$

etc. From (3.16) one can determine $b_{1}$ in terms of $a$ and $\alpha$; knowledge of $b_{1}$ yields $b_{2}$ in terms of $a$ and $\alpha$. In a similar way we can obtain the higher-order coefficients. The most important feature is that from Eq. (i) $C$ can be determined, i.e.,

$$
C=\bar{m}\left(\bar{\mu}_{0}-1\right) \text {. }
$$

We shall later show that

$$
C=-\frac{1}{2 \pi} \log \left|z_{0}\right|=-\frac{1}{2 \pi} \log \alpha .
$$

Thus (3.15) indeed measures the locations of the source and assigns the origin. Now, knowing the origin, we place the source at $z_{0}=i \alpha$, i.e. on the $y$ axis (see Fig. 4). In this case, from (3.9),

$$
\psi(z)=\frac{\partial}{\partial n}\left\{\frac{1}{2 \pi} \log |f(z)|+\frac{\text { I.P. }}{2 \pi a \alpha}\left(f(z)+\frac{1}{f(z)}\right)\right\}
$$




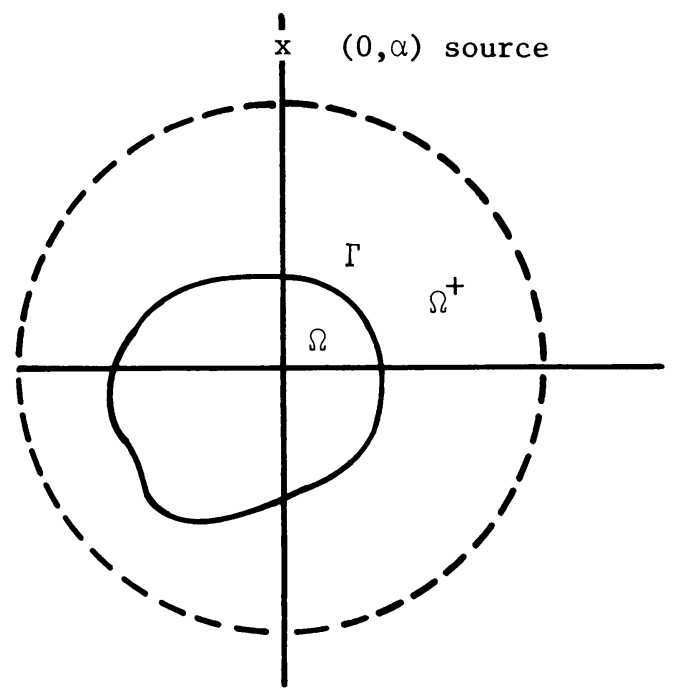

Fig. 4.

Therefore

$$
\tilde{\phi}(z)=\frac{1}{2 \pi a \alpha} \text { I.P. } \frac{\partial}{\partial n}\left(f(z)+\frac{1}{f(z)}\right)+\left(\frac{C}{\bar{m}}+1\right) \frac{1}{2 \pi} \frac{\partial}{\partial n} \log |f(z)| .
$$

Now the step: (3.11) through (3.16) remain valid. To show this let us denote the far-field coefficient co. esponding to (3.11) by $\mu_{n}^{*}$. Then

$$
\begin{array}{rl}
\bar{\mu}_{n}^{*}=-\frac{1}{2 \pi a \alpha} \int_{\Gamma} \text { I.P. } \frac{\partial}{\partial n}\left(f(z)+\frac{1}{f(z)}\right) z^{n} & d s \\
& -\frac{1}{2 \pi}\left(\frac{C}{\bar{m}}+1\right) \int_{\Gamma} \frac{\partial}{\partial n} \log |f(z)| z^{n} d s .
\end{array}
$$

The second integral in (3.20) remains the same as in (3.11). By the Cauchy-Riemann equations we observe that

but

$$
\begin{gathered}
\text { I.P. } \frac{\partial}{\partial n}\left(f(z)+\frac{1}{f(z)}\right)=\text { R.P. } \frac{\partial}{\partial s}\left(f(z)+\frac{1}{f(z)}\right), \\
\text { I.P. }\left(f(z)+\frac{1}{f(z)}\right)=0 \text { for } z \in \Gamma .
\end{gathered}
$$

Therefore

$$
\text { I.P. } \frac{\partial}{\partial n}\left(f(z)+\frac{1}{f(z)}\right)=\frac{\partial}{\partial s}\left(f(z)+\frac{1}{f(z)}\right),
$$

and when we substitute (3.21) into (3.20) we have

$$
\bar{\mu}_{n}^{*}=-\frac{1}{2 \pi a \alpha} \int_{|\omega|=1} \frac{\partial}{\partial \omega}\left(\omega+\frac{1}{\omega}\right)\left[f^{-1}(\omega)\right]^{n} d \omega+\frac{i}{2 \pi}\left(\frac{C}{\bar{m}}+1\right) \int_{\Gamma} \frac{1}{\omega}\left[f^{-1}(\omega)\right]^{n} d \omega .
$$


Computing the residues in (3.22) as before, we have

$$
\begin{gathered}
\bar{\mu}_{0}^{*}=-\left(\frac{C}{\bar{m}}+1\right), \\
\bar{\mu}_{1}^{*}=-\frac{i}{a \alpha}\left(-\frac{1}{a}+b_{1}\right) .
\end{gathered}
$$

Thus (3.16) and (3.24) are two equations for $a$ and $b_{1}$ in terms of known $\alpha, \bar{\mu}_{1}$ and $\bar{\mu}_{1}^{*}$. Adding these equations we have

$$
a^{2}=\frac{2 i}{\alpha\left(\bar{\mu}_{1}+\bar{\mu}_{1}^{*}\right)} .
$$

Thus the mapping radius $a$ can be determined.

Remark 3.2. Since in practice only a finite number of $a_{n}$ 's can be measured, we can determine only a finite number of $b_{n}$ 's in (3.14). In [2] an error estimate is given when (3.14) is truncated after $N$ terms. This applies in our case too.

Remark 3.3. In determining $\alpha$ we used the fact that

$$
C=-\frac{1}{2 \pi} \log \left|z_{0}\right| \text {. }
$$

If we consider the simple layer potential representation for the solution of problem II,

$$
v(\mathbf{x})=\frac{1}{2 \pi} \int_{\Gamma} \psi(y) \log |\mathbf{x}-\boldsymbol{y}| d s_{\mathbf{y}}+\frac{1}{2 \pi} \log \left|\mathbf{x}-\mathbf{x}_{0}\right|+C,
$$

for $|\mathbf{x}|<\left|\mathbf{x}_{0}\right|,\left(\mathbf{x} \in \Omega^{+}\right)$, Fourier expansion of $\log |\mathbf{x}-\mathbf{y}|$ gives no constant term in the integral. However,

$$
\frac{1}{2 \pi} \log \left|\mathbf{x}-\mathbf{x}_{0}\right|=\frac{1}{2 \pi} \log \left|z-z_{0}\right|=\frac{1}{2 \pi} \log \left|z_{0}\right|+\sum_{n=1}^{\infty} \frac{(-1)^{n}}{n}\left(\frac{z}{z_{0}}\right)^{n},
$$

which has a leading constant $(1 / 2 \pi) \log \left|z_{0}\right|$. Since $v(\mathbf{x})=0$ for $\mathbf{x} \in \Gamma, C+(1 / 2 \pi) \log \left|z_{0}\right|$ must be zero.

Remark 3.4. In our Laurent expansion (3.14) we took $b_{0}=0$. This was possible since the corresponding translation in the $z$ plane applied both to the scatterer and the source by the same amount. However, one can in fact calculate $b_{0}$ from Eqs. (3.13) and (3.20) [6].

\section{REFERENCES}

[1] D. L. Colton, The inverse scattering problem for a cylinder, Proc. Roy. Soc. Edinburgh A84, 135-143 (1979)

[2] D. L. Colton and R. Kleinman, The direct and inverse scattering problems for an arbitrary cylinder: Dirichlet boundary conditions, Proc. Roy. Soc. Edinburgh A86, 29-42 (1980)

[3] S. I. Hariharan and R. C. MacCamy, Integral equation procedures for eddy current problems, J. Computational Phys. (to appear).

[4] G. C. Hsiao and R. C. MacCamy, Solution of boundary value problems by integral equations of the first kind, SIAM Review 15, 687-705 (1973)

[5] S. I. Hariharan and E. Stephan, A boundary element method in two-dimensional electromagnetics, ICASE Report No. 81-14, April 28, 1981

[6] D. L. Colton, private communication 